\title{
BMJ Open Postural tachycardia syndrome is associated with significant symptoms and functional impairment predominantly affecting young women: a UK perspective
}

\author{
Claire McDonald, ${ }^{1}$ Sharon Koshi, ${ }^{1}$ Lorna Busner, ${ }^{2}$ Lesley Kavi, ${ }^{2}$ Julia L Newton ${ }^{1}$
}

To cite: McDonald C, Koshi S, Busner L, et al. Postural tachycardia syndrome is associated with significant symptoms and functional impairment predominantly affecting young women: a UK perspective. BMJ Open 2014:4:e04127. doi:10.1136/bmjopen-2013004127

- Prepublication history for this paper is available online. To view these files please visit the journal online (http://dx.doi.org/10.1136/ bmjopen-2013-004127).

Received 27 September 2013 Accepted 11 October 2013

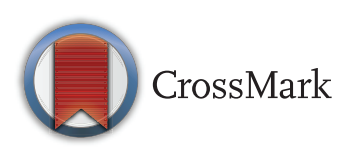

${ }^{1}$ Institute for Ageing \& Health, Campus for Ageing \& Vitality, Medical School, Newcastle University, Newcastle Hospitals NHS Foundation Trust, Newcastle upon Tyne, UK

${ }^{2}$ PoTs UK, www.potsuk.org

Correspondence to Professor Julia L Newton; J.L.newton@NCL.AC.UK

\section{ABSTRACT}

Objective: To examine a large UK cohort of patients with postural tachycardia syndrome (PoTS), to compare demographic characteristics, symptoms and treatment of PoTS at one centre compared to the largest patient group PoTS UK and to verify if their functional limitation is similar to patients with chronic fatigue syndrome (CFS).

Design: A cross-sectional study assessed the frequency of symptoms and their associated variables. Patients and setting: Two PoTS cohorts were: (1) recruited via PoTS UK, (2) diagnosed at Newcastle Hospitals National Health Service (NHS) Foundation Trust 2009-2012. Patients with PoTS were then compared to a matched cohort with CFS.

Main outcome measures: Patients' detailed demographics, time to diagnosis, education, disability, medications, comorbidity and precipitants. Symptom assessment tools captured, Fatigue Impact Scale, Epworth Sleepiness Scale, Orthostatic Grading Scale (OGS), Hospital Anxiety and Depression Scale, Health Assessment Questionnaire, Cognitive Failures Questionnaire.

Results: 136 patients with PoTS participated (84 members of PoTS UK (170 cohort; $50 \%$ return) and 52 (87 cohort; 60\%) from Newcastle Clinics). The PoTS UK population was significantly younger than the clinic patients, with significantly fewer men $(p=0.005)$. Over $60 \%$ had a university or postgraduate degree. Significantly more of the PoTS UK cohort were working, with hours worked being significantly higher $(p=0.001)$. Time to diagnosis was significantly longer in the PoTS UK cohort $(p=0.04)$. Symptom severity was comparable between cohorts. The PoTS total group was compared with a matched CFS cohort; despite comparable levels of fatigue and sleepiness, autonomic symptom burden (OGS) was statistically significantly higher. The most common treatment regime included $\beta$-blockers. Overall, 21 treatment combinations were described. Up to $1 / 3$ were taking no treatment.

Conclusions: Patients with PoTS are predominantly women, young, well educated and have significant and debilitating symptoms that impact significantly on

\section{Strengths and limitations of this study}

- Description of the demographics and symptom burden experienced by a large cohort of patients with positional tachycardia syndrome.

- Cross-sectional 'opt in' study using self-report symptom assessment tools.

- Study performed in collaboration with the national PoTS patient support group.

quality of life. Despite this, there is no consistent treatment.

\section{INTRODUCTION}

Postural tachycardia syndrome (PoTS) is a subset of orthostatic intolerance that is associated with the presence of excessive tachycardia on standing. Symptoms may be so severe that normal activities of life, such as bathing, housework and even eating, can be significantly limited. ${ }^{1}$ Patients with PoTS have been reported to suffer from a degree of functional impairment similar to that seen in conditions such as chronic obstructive pulmonary disease and congestive heart failure; yet these patients are frequently misdiagnosed as having severe anxiety, panic disorder or chronic fatigue syndrome (CFS) because of a lack of awareness of the condition and its functional impact by healthcare professionals. $^{2}$

Studies performed in the USA suggest that PoTS affects approximately 170/100 000 of the population, and of this total, $25 \%$ are disabled and unable to work. PoTS can affect any age group, but predominantly presents in young and middle age groups. ${ }^{3}$ No such studies currently exist from the UK. 
The principal feature of PoTS is orthostatic intolerance. This is defined as the provocation of symptoms on standing that are relieved by lying down. ${ }^{4}$ Those with PoTS frequently present with palpitations, fatigue, lightheadedness, exercise intolerance, nausea, diminished concentration, tremulousness, syncope and presyncope. ${ }^{5}$

In adults, PoTS is currently defined as the presence of symptoms of orthostatic intolerance associated with a heart rate increase of $30 \mathrm{bpm}$ (or a rate that exceeds $120 \mathrm{bpm}$ ) that occurs within the first $10 \mathrm{~min}$ of standing or upright tilt, not associated with other chronic debilitating conditions such as prolonged bed rest or the use of medications known to diminish vascular or autonomic tone. ${ }^{6}$ In teenagers, the criteria suggest that an increase in $40 \mathrm{bpm}$ on standing is diagnostic. ${ }^{7}$ The aim of this current study was twofold. First, we wanted to raise awareness of the prevalence and impact of PoTS in healthcare professionals by collating a large cohort of patients with PoTS in the UK. Second, we wanted to see whether there were differences between patients with PoTS seen in one centre compared with members of the largest patient support group PoTS UK who provide support exclusively for patients with PoTS.

\section{METHODS}

\section{Patients}

\section{Postural tachycardia syndrome}

Two cohorts of patients with PoTS were included in this study; the first cohort was recruited via the national patient support group PoTS UK (http://www.potsuk. org.uk) and the second cohort was made up of all those diagnosed with PoTS at the Falls and Syncope Service Newcastle upon Tyne Hospitals National Health Service (NHS) Foundation Trust between May 2009 and April 2012. Both cohorts included only patients where a diagnosis had been reached by a secondary care clinician. PoTS UK is an independent UK registered small charity. People join PoTS UK after finding the website through search engines or links from other websites. At the time of this study, PoTS UK had 170 members. The Newcastle Clinic Cohort comprised 87 patients with PoTS.

\section{Patients with CFS}

Patients with CFS were consecutive patients referred to the Newcastle Royal Victoria Infirmary who fulfilled the Fukuda 1994 diagnostic criteria for $\mathrm{CFS}^{8}$ who had been shown on haemodynamic testing to not have PoTS.

\section{Symptom assessment tools}

The complete survey form was developed in two parts. The first part contained questions about demographics, age since symptoms began and age at diagnosis, education, disability, medications and symptoms at the time of completing the survey. Next were questions about characteristics of PoTS including comorbidity and potential precipitants. Following these, participants completed six validated symptom assessment tools quantifying fatigue, daytime sleepiness, orthostatic symptoms, anxiety and depressive symptoms, functional ability and cognitive failures.

\section{Fatigue Impact Scale}

The Fatigue Impact Scale (FIS) assesses perceptions of how fatigue affects cognitive, physical and psychosocial functions. It is validated for self-completion and used in a number of fatigue-associated diseases ${ }^{9-13}$ and CFS. ${ }^{13}$ It comprises 40 items; participants rate how these items are affected by fatigue on a five-point scale: 0 (no problem) to 4 (extreme problem). Total score is calculated by adding responses from the 40 questions; possible range $0-160$.

\section{Epworth Sleepiness Scale}

A validated self-report assessment tool that quantifies symptoms of daytime sleepiness. ${ }^{14}$ Consists of eight items, each graded $0-3$. Higher scores indicate a greater impact of daytime sleepiness.

\section{Orthostatic Grading Scale}

Quantifies the symptoms of orthostatic intolerance due to hypotension, with questions related to frequency and severity of symptoms and interference with daily activities. ${ }^{15}$ It consists of five items, each graded on a scale of $0-4$. Higher scores indicate a greater impact of orthostatic symptoms.

\section{Hospital Anxiety and Depression Scale}

A validated anxiety and depression measure optimised for use in patients with chronic disease. ${ }^{16}$ Individual subscales comprise seven items, each with a potential score 0-21. For the purposes of this study, 'caseness' for depression or anxiety was defined as a score of 11 or greater for the subscale.

\section{Patient-Reported Outcomes Measurement Information System, Health Assessment Questionnaire (PHAQ)}

Assesses the functional and physical ability of participants. ${ }^{17}$ The PHAQ consists of 20 items that ask patients to rate their ability to carry out daily activities on a fivepoint scale of ' $0=$ without any difficulty' to ' $4=$ unable to do'. Higher scores indicate a worse functional ability and therefore greater functional impairment.

\section{Cognitive Failures Questionnaire}

Assesses cognitive symptom prevalence by measuring the frequency of cognitive slips or failures occurring in everyday life. ${ }^{18}$ Consists of 25 items covering failures in perception, memory and motor function and asks patients to rate how often these failures occur, on a fivepoint Likert scale $0-4 \quad(0=$ never, $4=$ very often $)$. The responses for the 25 questions are added together to obtain the total Cognitive Failures Questionnaire (CFQ) score. The higher the score, the greater the cognitive impairment. 


\section{Study design}

We used a cross-sectional study design to assess the frequency of symptoms and their associated variables at a single point in time. The same survey was sent to all members of PoTS UK and PoTS clinic patients in 2012. Patients who were 16 years old or older were requested to complete and return the survey. For the PoTS UK cohort, surveys were emailed by PoTS UK to all members. The survey was featured on the PoTS UK website and in the newsletter. No participant identifiable information was provided to the investigators, and therefore no written consent was obtained from participants but return of the anonymous questionnaires was considered as implied consent for the use of the data. For the PoTS clinic cohort, surveys were mailed to all patients who had been diagnosed with PoTS. To increase response, surveys were mailed one additional time to those who had not returned the initial survey within 4 weeks. Patients in the Falls and Syncope Service provide written informed consent agreeing that they are happy to participate in research, audit and service evaluation. Results of the symptom assessment tools were compared with those completed by a cohort of matched patients with CFS attending our clinic.

\section{Analysis}

All analyses were carried out using statistical software (GraphPad, Prism V.5). Descriptive statistics for each of the analysed parameters were initially calculated separately for the PoTS UK and the clinic cohorts. For continuous parameters, either median and range, or mean and SE, were calculated; for categorical variables, percentages were calculated.

\section{RESULTS}

\section{Response rates}

A total of 136 patients with PoTS completed and returned the questionnaires. This comprised 84 members of PoTS UK (170 cohort; $50 \%$ return) and 52 $(87$ cohort; $60 \%)$ ) patients with PoTS attending the Falls and Syncope Service.

\section{Demographics of the two groups}

The PoTS UK population was significantly younger than the PoTS clinic patients, with significantly fewer men ( $p=0.005$; table 1$)$. Interestingly, there were proportionately more smokers in the PoTS UK group but relatively similar proportions drinking alcohol and taking caffeine. There were, however, four in the PoTS UK population (5\%) compared to none in the PoTS clinic cohort who were taking recreational drugs specifically stated to be cannabis. On reviewing the educational level of both cohorts, it became clear that, taken together, patients with PoTS are well educated, with over $60 \%$ of the population having either a university or a postgraduate degree. This was comparable between the two cohorts. Significantly more of the PoTS UK cohort were working and when they were working, the hours that they were able to work were significantly higher $(\mathrm{p}=0.001)$. Despite this, a large proportion of the patients with PoTS had to change their job because of ill health and had been unable to work because of problems with their health.

\section{Overall symptom burden of the groups}

Symptoms started at a significantly younger age in the PoTS UK cohort (23 vs 28 years of age) and diagnosis age was not significantly different with the PoTS UK population being diagnosed on average at the age of 30 compared with 33 in the PoTS clinic cohort. As a consequence, time to diagnosis after the onset of symptoms was significantly longer in the PoTS UK population compared with the group attending a specialist clinic $(\mathrm{p}=0.04)$. When considering the symptom assessment tools completed by participants with PoTS, there were no significant differences in symptom severity between the PoTS UK cohort and PoTS clinic cohort, with high levels of fatigue, daytime sleepiness, orthostatic symptoms, anxiety and depression, particularly high levels of functional impairment and cognitive symptoms.

Around $20 \%$ of the total cohort reported having CFS and just under $20 \%$ had Ehlers-Danlos syndrome, but when we considered the PoTS UK population compared with the PoTS clinic cohort, this showed some interesting differences, with the PoTS clinic cohort having $42 \%$ of the cohort with comorbid CFS/myalgic encephalomyelitis (ME) compared with 26\% of the PoTS UK having comorbid Ehlers-Danlos syndrome (EDS; table 1).

\section{Comparing the total PoTS group to a matched CFS group}

In view of the fact that the PoTS UK and the PoTS clinic cohort were comparable in terms of symptom severity, we considered the total group $(\mathrm{n}=136)$ and compared this to an age-matched and sex-matched cohort of patients with CFS. The CFS group had a mean \pm SD age of $36 \pm 10$ years $(p=n s)$. Interestingly, despite comparable levels of symptom burden measured by the FIS and daytime sleepiness measured by the Epworth Sleepiness Scale, the scores from the Orthostatic Grading Scale (OGS; autonomic symptom burden) were statistically significantly higher in the PoTS group compared with the CFS group. Hospital Anxiety and Depression Scale (HADS) scores were significantly higher in the CFS group despite high levels of symptoms in both groups (figure 1).

\section{Comparing the symptom burden of patients with PoTS with and without comorbid CFS/ME}

We went on to consider the total PoTS cohort of 136 patients and compared those with $(\mathrm{n}=27)$ and without $(n=109)$ comorbid CFS. When we considered the number of symptoms that they first noticed before being diagnosed with PoTS, those without CFS had significantly more symptoms $(p=0.0004)$, with the majority of these symptoms being palpitations, dizziness, memory 
Table 1 Demographics of the PoTS UK cohort compared with the PoTS clinic cohort

\begin{tabular}{|c|c|c|c|c|}
\hline & PoTS UK & PoTS clinic & p Value & Total PoTS \\
\hline $\mathrm{N}$ & 84 & 52 & & 136 \\
\hline Age, mean $\pm S D$ & $32 \pm 10$ & $36 \pm 11$ & 0.04 & $33 \pm 10$ \\
\hline Males, n (\%) & $3(4)$ & $10(19)$ & 0.005 & $13(10)$ \\
\hline BMI, mean $\pm S D$ & $24 \pm 5$ & $34 \pm 5$ & 0.09 & $27 \pm 5$ \\
\hline Smokers, n (\%) & $12(14)$ & $2(4)$ & 0.08 & $14(10)$ \\
\hline Previous smoker, n (\%) & $14(17)$ & $6(12)$ & 0.5 & $20(15)$ \\
\hline Alcohol, yes, n (\%) & $28(33)$ & $20(38)$ & 0.6 & $48(35)$ \\
\hline Caffeine, yes, n (\%) & $46(55)$ & $34(65)$ & 0.3 & $80(59)$ \\
\hline Drugs, yes, n (\%) & $4(5)$ & 0 & 0.3 & $4(3)$ \\
\hline Age symptoms started, mean \pm SD & $23 \pm 10 n=76$ & $28 \pm 12 n=50$ & 0.01 & $24 \pm 11$ \\
\hline Age diagnosed, mean $\pm S D$ & $30 \pm 10 n=82$ & $33 \pm 11 n=48$ & 0.09 & $31 \pm 10$ \\
\hline Difference, mean $\pm S D$ & $8.3 \pm 10 n=79$ & $5.2 \pm 5 n=49$ & 0.04 & $6.7 \pm 7$ \\
\hline \multicolumn{5}{|l|}{ Symptoms assessment tools } \\
\hline Fatigue Impact Scale & $91 \pm 32$ & $92 \pm 37$ & 0.8 & $92 \pm 34$ \\
\hline Epworth Sleepiness Scale & $10 \pm 5$ & $10 \pm 8$ & 0.8 & $10 \pm 5$ \\
\hline Orthostatic Grading Scale & $13 \pm 4$ & $12 \pm 4$ & 0.4 & $13 \pm 4$ \\
\hline Hospital Anxiety and Depression & $14 \pm 7$ & $15 \pm 9$ & 0.3 & $15 \pm 7$ \\
\hline $\begin{array}{l}\text { Patient Reported Outcome Measure Health } \\
\text { Assessment Questionnaire }\end{array}$ & $26 \pm 21$ & $26 \pm 22$ & 1.0 & $26 \pm 21$ \\
\hline Cognitive Failures Questionnaire & $50 \pm 24$ & $51 \pm 23$ & 0.7 & $51 \pm 23$ \\
\hline \multicolumn{5}{|l|}{ Comorbidity } \\
\hline Chronic Fatigue Syndrome & $6(7)$ & $22(42)$ & 0.0001 & $28(21)$ \\
\hline Ehlers Danlos Syndrome & $22(26)$ & $2(4)$ & 0.0009 & $24(18)$ \\
\hline Irritable Bowel Syndrome & $9(11)$ & $5(10)$ & 1.0 & $14(10)$ \\
\hline Thyroid & $10(12)$ & $3(6)$ & 0.4 & $13(10)$ \\
\hline Heart & $4(5)$ & 0 & 0.3 & $4(3)$ \\
\hline Fibromyalgia & $7(8)$ & $2(4)$ & 0.5 & $9(7)$ \\
\hline \multicolumn{5}{|l|}{ Education } \\
\hline Left school at 16 & $9(11)$ & $12(23)$ & 0.09 & $21(15)$ \\
\hline Left school at 18 & $7(8)$ & $5(10)$ & 1.0 & $12(9)$ \\
\hline University degree & $33(40)$ & $23(44)$ & 0.6 & $56(41)$ \\
\hline Postgraduate degree & $16(19)$ & $12(23)$ & 0.7 & $28(21)$ \\
\hline Other & $14(17)$ & 0 & & $14(10)$ \\
\hline
\end{tabular}

impairment, breathlessness, light-headedness and muscle aches. Twenty-three $(21 \%)$ of the patients with PoTS and without CFS were currently receiving disability benefit compared with $13(48 \%)$ of the patients with PoTS and CFS (table 2). The participants with PoTS with no CFS appeared to be more able to exercise, with $44 \%$ of the patients with PoTS with no CFS currently engaging in any form of exercise compared with $29 \%$ of the patients with PoTS and with CFS. In those who were not exercising, the vast majority in both groups felt that this was because of fatigue or because they knew it would worsen their symptoms. In terms of hours that they had spent performing activities, there was no significant difference between patients with PoTS with and without CFS in terms of household-related activities, social and recreational activities and family-related activities; however, the PoTS without CFS group did appear to have spent significantly more hours in work-related activities. Interestingly, 34\% of the PoTS without CFS group had spontaneously reported episodes of loss of consciousness in association with their symptoms compared with $18 \%$ of the PoTS with CFS group. When we reviewed symptoms in the two groups that would be consistent with the diagnosis of CFS (Fukuda), of patients with PoTS who did not describe comorbid CFS, $43 \%$ of them would actually have met the diagnostic criteria for CFS. The vast majority of patients with PoTS had noticed an infection as the precipitating cause for their symptoms, and interestingly, six of the PoTS without CFS group (seven in total) did not describe themselves as being ill at all. Scores from the symptom assessment tools confirmed that those with PoTS with and without CFS had higher levels of orthostatic intolerance measured using the OGS compared with the CFS group (figure 2).

\section{The impact of treatments}

The treatments that patients with PoTS were using are shown in table 3. The most common treatment, either alone or in combination, was $\beta$-blockers. Overall, there was a wide range of different therapies being used with 21 different combinations described by patients with PoTS. In total, $26.5 \%$ of patients in PoTS UK and $34.5 \%$ 
Figure 1 Symptom assessment tools in the total postural tachycardia syndrome (PoTS) group compared with the age-matched and sex-matched chronic fatigue syndrome (CFS) group. Autonomic symptom burden and scores from Hospital Anxiety and Depression Scale are higher in the PoTS group compared with the CFS group while fatigue and daytime sleepiness are comparable.
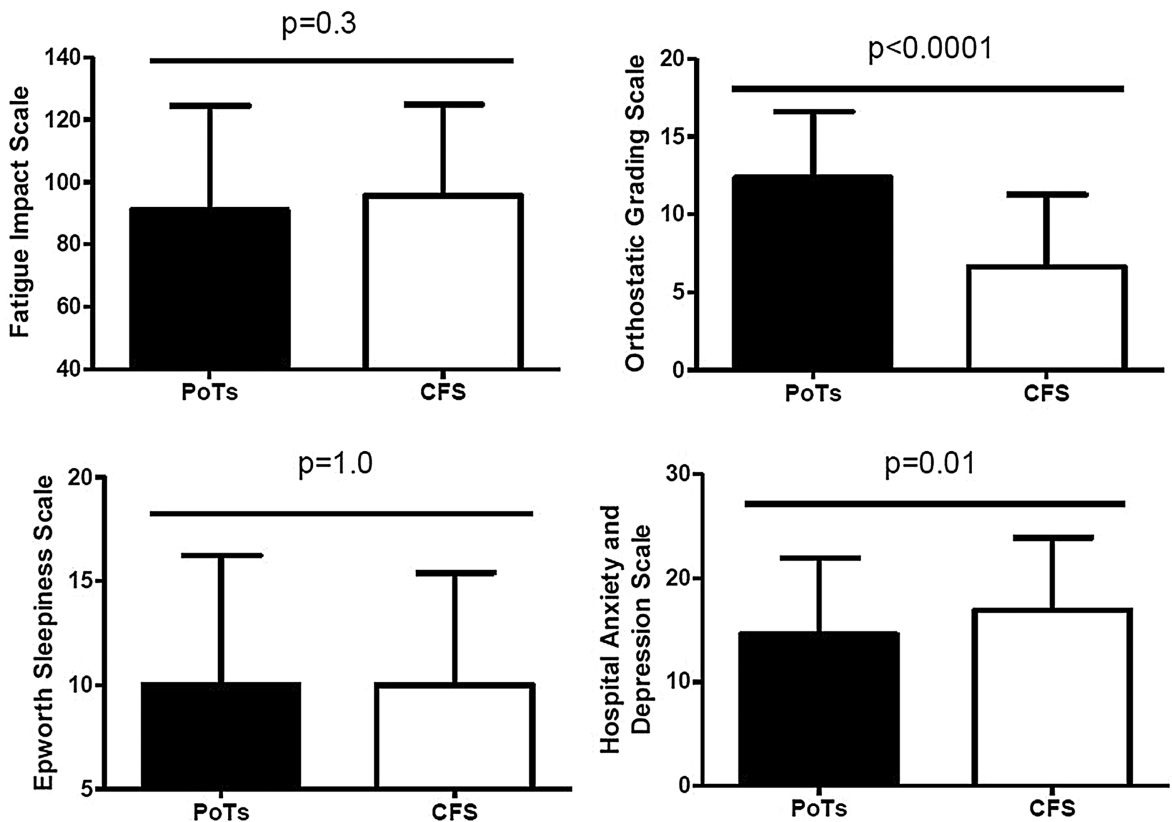

Table 2 Comparison between the postural tachycardia syndrome (PoTS) with comorbid chronic fatigue syndrome (CFS) and POTS without comorbid CFS

If you do not exercise, why do you not exercise?

$\mathrm{n}(\%)$

In the past 4 weeks, how many hours have you spent (median hours/week (IQR)) Other symptoms ( $\mathrm{n}$ (\%)) Onset (n (\%))
Proportion working n (\%)

When working hours worked (median (IQR))

Number of symptoms first noticed (median (IQR))

Palpitations n (\%)

Fatigue $\mathrm{n}(\%)$

Dizziness $n(\%)$

Memory $n(\%)$

Breathlessness $\mathrm{n}(\%)$

Light-headedness n (\%)

Muscle aches $n(\%)$

Are you currently engaging in any form of exercise? $\mathrm{n}(\%)$

Not interested

No time

Would like to but cannot because of fatigue

Cannot because symptoms worsen

Household-related activities

Social and recreational activities

Family-related activities

Work-related activities

Reports LOC or presyncope

Symptoms consistent with CFS

Infection

Accident

Holiday

Immunisation

Surgery

Severe stress

Other

I am not ill

\begin{tabular}{|c|c|c|}
\hline $\begin{array}{l}\text { PoTS } \\
\text { No CFS }\end{array}$ & $\begin{array}{l}\text { PoTS } \\
\text { With CFS }\end{array}$ & p Value \\
\hline 109 & 27 & \\
\hline $57(52)$ & $11(41)$ & 0.4 \\
\hline $30(26-33)$ & $5(0.2-23)$ & 0.001 \\
\hline $2(2.3-2.9)$ & $1(1.1-2.3)$ & 0.0004 \\
\hline $80(73)$ & $16(60)$ & 0.9 \\
\hline 85 (78) & 24 (89) & \\
\hline 87 (80) & $20(74)$ & \\
\hline 47 (43) & $13(48)$ & \\
\hline $63(58)$ & $14(52)$ & \\
\hline $89(82)$ & $20(74)$ & \\
\hline $54(50)$ & $16(59)$ & \\
\hline 48/109 (44) & $8 / 27(30)$ & 0.2 \\
\hline $1 / 61(2)$ & $0 / 20$ & 1.0 \\
\hline $7 / 61$ (12) & $0 / 20$ & \\
\hline 46/61 (75) & $16 / 20(80)$ & \\
\hline 49/61 (80) & $17 / 20(85)$ & \\
\hline $5(6-11)$ & $4(4-16)$ & 0.5 \\
\hline $4(4-7)$ & $4(3-7)$ & 0.8 \\
\hline $5(6-13)$ & $5(4-12)$ & 1.0 \\
\hline $3(11-20)$ & $0(0.8-12)$ & 0.04 \\
\hline $37 / 109$ (34) & $5 / 27$ (19) & 0.2 \\
\hline 47/109 (43) & 20/27 (74) & 0.005 \\
\hline 35/109 (32) & $16 / 27$ (59) & 0.05 \\
\hline $14 / 109$ (13) & $1 / 27$ (4) & \\
\hline $7 / 109(6)$ & $1 / 27(4)$ & \\
\hline 6/109 (6) & $7 / 27$ (26) & \\
\hline 10/109 (9) & 4/27 (15) & \\
\hline 24/109 (22) & $8 / 27$ (30) & \\
\hline $34 / 109$ (31) & 5/27 (19) & \\
\hline $6 / 109(6)$ & $1 / 27(4)$ & \\
\hline
\end{tabular}

LOC, loss of consciousness. 


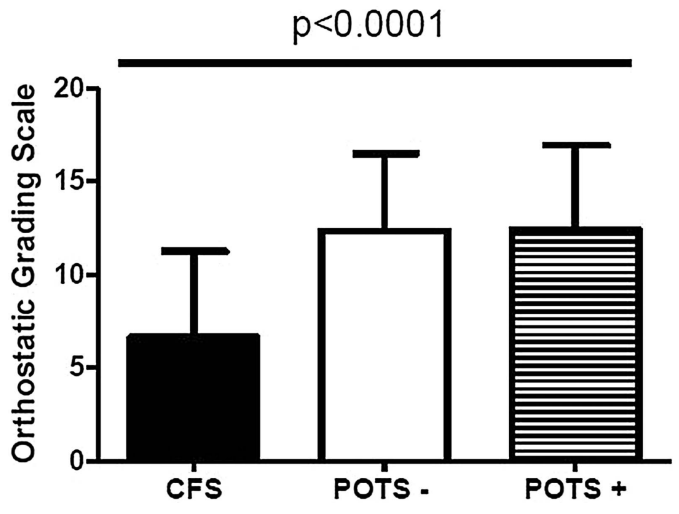

Figure 2 Orthostatic Grading Scale scores in the postural tachycardia syndrome (PoTS) group with comorbid chronic fatigue syndrome (CFS; PoTS +) and PoTS without comorbid CFS (PoTS -) are significantly higher compared with the group with CFS.

in the PoTS clinic cohort were taking no treatment or only salt for their PoTS. When we compared those taking medication to those not, there were no significant differences in fatigue severity, daytime sleepiness, cognitive or autonomic symptom burden, and nor was the level of functional impairment different (data not shown). Those taking no medication did, however, score higher on HADS compared with those taking medication (mean \pm SD HAD $17 \pm 8$ vs $14 \pm 7 ; \mathrm{p}=0.01$ ).

Table 3 Medications used by patients with postural tachycardia syndrome (PoTS)

\begin{tabular}{llll}
\hline $\begin{array}{l}\text { Primary } \\
\text { treatment }\end{array}$ & Combination & PoTS UK & $\begin{array}{l}\text { PoTS } \\
\text { clinic }\end{array}$ \\
\hline No treatment & & $20(24 \%)$ & $17(32.5 \%)$ \\
$\beta$-blocker & Alone & $15(18 \%)$ & $10(19 \%)$ \\
& Plus midodrine & $4(5 \%)$ & $2(4 \%)$ \\
Ivabradine & Alone & $4(5 \%)$ & $7(13 \%)$ \\
& Midodrine & 0 & $2(4 \%)$ \\
& Fludrocortisone & $1(1 \%)$ & $1(2 \%)$ \\
& Sertraline & $1(1 \%)$ & 0 \\
& Duloxetine & $1(1 \%)$ & 0 \\
Diltiazem & p-blocker & 0 & $1(2 \%)$ \\
& Alone & & $1(2 \%)$ \\
Verapamil & Fludrocortisone & $1(1 \%)$ & 0 \\
Fludrocortisone & Midodrine & 0 & $1(2 \%)$ \\
& Alone & 0 & $1(2 \%)$ \\
& Alone & $5(6 \%)$ & $2(4 \%)$ \\
Midodrine & M-blocker & $5(6 \%)$ & $2(4 \%)$ \\
& Midodrine & $5(6 \%)$ & 0 \\
Octretide & Alone & $12(14 \%)$ & $4(7.5 \%)$ \\
& Buproprion & $1(1 \%)$ & 0 \\
Pregabalin & + midodrine & $2(2.5 \%)$ & 0 \\
Clonazepam & + fludro & & \\
Antidepressant & & $1(1 \%)$ & 0 \\
Salt only & & $1(1 \%)$ & 0 \\
\hline & & $3(4 \%)$ & 0 \\
& & $2(2.5 \%)$ & $1(2 \%)$ \\
\hline & & &
\end{tabular}

\section{DISCUSSION}

In this study, we have explored for the first time the characteristics of patients with PoTS in the UK using a clinic-based cohort and a population obtained via a national patient support group. We have shown that patients with PoTS are predominantly women, young, well educated and have significant and debilitating symptoms that impact significantly on their quality of life. Despite this, there is no consistent treatment, high levels of disability and associated comorbidity.

Importantly for patients, we found that although individuals presented with symptoms at the same age, those attending a specialist clinic received a diagnosis quicker. We would suggest that this advocates the need for specialist care for those with PoTS to reduce the overall impact of the condition. The average age of diagnosis was young; however, there was a wide age range with the oldest patients with PoTS presenting at age 63. It is therefore vital that the symptoms are recognised, taken seriously and treated early.

The differences between a cohort from one clinic and the national patient support service were interesting. Most of the current PoTS literature comes from one or more specialist referral clinics. The data from our survey reflect the broader population with PoTS and may therefore be different from the experiences that are usually reported in the literature.

The symptom burden for those with PoTS is high and we have shown it to be comparable to that seen in CFS. CFS is a chronic condition that is recognised by the WHO as a neurological disorder and by the Disability Discrimination Act 2005 as a disability. ${ }^{19}$ We would suggest that our data highlight that at the current time those with PoTS suffer to the same extent as those with CFS but do not receive the same protection from the law. Our study confirming that patients with PoTS had even higher autonomic symptom scores measured using the OGS might explain in part the high prevalence of dysautonomia in unselected cohorts of patients with CFS. ${ }^{20-22}$ It is important that more work is carried out to understand the underlying autonomic abnormality in those with PoTS in order to allow us to develop targeted treatments that are effective and go beyond the currently available simply symptomatic management.

Our finding of high levels of comorbidity, particularly CFS and EDS, is important as it might point towards an underlying overlapping mechanism. ${ }^{23-25}$ We believe that more work is needed to understand this spectrum of diseases and whether lessons could be learnt from one disease that could be applied to others. It is possible that the higher prevalence of CFS reported in the PoTS clinic cohort reflects the research interests of the clinician and therefore a referral bias. However, the lower prevalence of EDS in the clinic cohort has highlighted important training needs for the Newcastle clinicians and it will be interesting to explore in the future whether the prevalence rates of EDS in patients with PoTS attending the Newcastle clinic increase. 
This study has a number of limitations. The prevalence of CFS and EDS was determined by self-report and therefore could be considered to be unreliable. Further studies are needed that involve clinical characterisation of patients with PoTS to determine directly whether selfreports are accurate. The response rates were low, particularly from the patients' support group PoTS UK, something which was surprising considering the lack of research and evidence with this group of patients and the strong desire for a greater understanding. It is possible that the membership of PoTS UK, which is in excess of 170, is comprised of sufferers but also carers and relatives.

Interestingly, $24 \%$ of patients with PoTS from PoTS UK and $33 \%$ of the clinic cohort were taking no medication for their PoTS. This was a surprise to us and we would suggest that it is an important observation for clinicians managing patients, that is, medication might not always be the answer, but it might also be that the current available treatments are associated with high levels of unacceptable side effects that are not tolerated by patients with PoTS. This, together with the 21 different treatment regimes described, strongly underlines the need for more evidence regarding treatments in PoTS and the urgent need for randomised controlled trials. This is similar to the findings from the largest published US series from the Mayo Clinic. ${ }^{26}$ The most frequently used medication appears to be $\beta$-blockers, which provide symptomatic control of the heart rate. With a better understanding of the characteristics of patients with PoTS and the associated comorbidity and autonomic abnormalities present on objective testing, it is hoped that better treatments can be developed. These treatments will need to not only provide symptomatic relief but also look at reducing the perpetuation of symptoms on those affected acutely and perhaps even stopping establishment of PoTS after the acute precipitating event.

It is becoming increasingly clear that historically many patients with PoTS were given a diagnosis of CFS/ME. A recent cross-sectional study performed by us has confirmed that $27 \%$ of those with a diagnosis of CFS have PoTS. $^{27}$ This is important when the public health implications of CFS/ME, and fatigue in its more general sense, are considered.

At present, only limited data are available on the prognosis of patients with PoTS. One recent retrospective study has suggested that the outcome is benign, ${ }^{28}$ although we would argue that our study suggests that the effects that PoTS has for individuals affected is far from benign and is associated with considerable morbidity. Our experience suggests that some patients never recover, and that a subset will worsen over time.

In conclusion, PoTS is a condition that is associated with significant symptoms that impact on quality of life. Currently, there are no evidence-based treatments for PoTS, and its underlying pathogenesis, natural history and associated features are not fully understood. We would suggest that increasing awareness of this debilitating disease is important to improve understanding, diagnosis and management of PoTS.

Contributors JLN and CM developed the idea, delivered the project, analysed the data and wrote the first draft of the paper. SK delivered the project and provided some analysis of the data. LB and LK coordinated the distribution of surveys to members of PoTS UK.

Funding Funding was provided for the study by the UK NIHR Biomedical Research Centre in Aging.

Competing interests None.

Ethics approval Newcastle Hospitals NHS Foundation Trust.

Provenance and peer review Not commissioned; externally peer reviewed.

Data sharing statement Additional unpublished anonymised data can be made available to those who approach the main authors.

Open Access This is an Open Access article distributed in accordance with the Creative Commons Attribution Non Commercial (CC BY-NC 3.0) license, which permits others to distribute, remix, adapt, build upon this work noncommercially, and license their derivative works on different terms, provided the original work is properly cited and the use is non-commercial. See: http:// creativecommons.org/licenses/by-nc/3.0/

\section{REFERENCES}

1. Grubb BP. Postural orthostatic tachycardia syndrome. Circulation 2008;117:2814-17.

2. Grubb BP, Row $P$, Calkins $H$. Postural tachycardia, orthostatic intolerance and the chronic fatigue syndrome. In: Grubb BP, Olshansky B, eds. Syncope: mechanisms and management. 2nd edn. Malden, MA: Blackwell/Future Press, 2005:225-44.

3. Schondorf R, Low PA. Idiopathic postural tachycardia syndrome. Ann Neurol 1990;28:271.

4. Low P, Opfer-Gehrking T, Textor S, et al. Postural tachycardia syndrome (POTS). Neurology 1995;45:519-25.

5. Karas B, Grubb BP, Boehm K, et al. The postural tachycardia syndrome: a potentially treatable cause of chronic fatigue, exercise intolerance and cognitive impairment. Pacing Clin Electrophysiol 2000;22:344-51.

6. Grubb BP, Kanjwal Y, Kosinski D. The postural tachycardia syndrome: a concise guide to diagnosis and management. J Cardiovasc Electrophysiol 2006;14:108-12.

7. Freeman R, Wieling W, Axelrod FB, et al. Consensus statement on the definition of orthostatic hypotension, neurally mediated syncope and the postural tachycardia syndrome. Clin Auton Res 2011;21:69-72.

8. Fukuda K, Straus SE, Hickie I, et al. International chronic fatigue syndrome study group. The chronic fatigue syndrome: a comprehensive approach to its definition and study. Ann Int Med 1994;121:953-9.

9. Fisk JD, Ritvo PG, Ross L, et al. Measuring the functional impact of fatigue: initial validation of the fatigue impact scale. Clin Infect Dis 1994;18:S79-83.

10. Prince MI, James OF, Holland NP, et al. Validation of a fatigue impact score in primary biliary cirrhosis: towards a standard for clinical and trial use. J Hepatol 2000;32:368-73.

11. Jones DE, Bhala N, Burt J, et al. Four year follow up of fatigue in a geographically defined primary biliary cirrhosis patient cohort. Gut 2006;55:536-41.

12. Jones DE, Gray JC, Newton J. Perceived fatigue is comparable between different disease groups. QJM 2009;102:617-24.

13. Newton JL, Okonkwo O, Sutcliffe K, et al. Symptoms of autonomic dysfunction in chronic fatigue syndrome. QJM 2007;100:519-26.

14. Johns M. Sleepiness in different situations measured by the Epworth Sleepiness Scale. Sleep 1994;17:703-10.

15. Schrezenmaier C, Gehrking JA, Hines SM, et al. Evaluation of orthostatic hypotension: relationship of a new self-report instrument to laboratory-based measures. Mayo Clin Proc 2005;80:330-3.

16. Zigmond AS, Snaith RP. The hospital anxiety and depression scale. Acta Psychiatr Scand 1983;67:361-70.

17. Bruce B, Fries JF. The Stanford health assessment questionnaire (HAQ): a review of its history, issues, progress, and documentation. J Rheumatol 2003;30:167-78. 
18. Broadbent DE, Cooper PF, FitzGerald P, et al. The Cognitive Failures Questionnaire (CFQ) and its correlates. Br J Clin Psychol 1982;21:1-16.

19. Chronic Fatigue Syndrome/Myalgic Encephalomyelitis (encephalopathy); diagnosis and management. http://www.nice.gov. org (accessed Mar 2013).

20. Rowe PC, Calkins H. Neurally mediated hypotension and chronic fatigue syndrome. Am J Med 1998;105:15S-21S.

21. Schondorf $R$, Freeman $R$. The importance of orthostatic intolerance in the chronic fatigue syndrome. Am J Med Sci 1999;317:117-23.

22. Schondorf R, Benoit J, Wein T, et al. Orthostatic intolerance in the chronic fatigue syndrome. J Auton Nerv Syst 1999;75:192-201.

23. Nijs J, Aerts A, De Meirleir K. Generalized joint hypermobility is more common in chronic fatigue syndrome than in healthy control subjects. J Manipulative Physiol Ther 2006;29:32-9.
24. Barron DF, Cohen BA, Geraghty MT, et al. Joint hypermobility is more common in children with chronic fatigue syndrome than in healthy controls. J Pediatr 2002;141:421-5.

25. Rowe PC, Barron DF, Calkins H, et al. Orthostatic intolerance and CFS associated with Ehlers-Danlos syndrome. J Pediatr 1999;135:494-9.

26. Thieben MJ, Sandroni P, Sletten DM, et al. Postural orthostatic tachycardia syndrome: the Mayo Clinic experience. Mayo Clin Proc 2007:82:308-13.

27. Hoad A, Spickett G, Elliott J, et al. Postural orthostatic tachycardia syndrome is an under-recognized condition in chronic fatigue syndrome. QJM 2008;101:961-5.

28. Sousa A, Lebreiro A, Freitas J, et al. Long-term follow-up of patients with postural tachycardia syndrome. Clin Auton Res 2012;22:151-3. 